American Medical Journal 3 (1): 50-55, 2012

ISSN 1949-0070

(C) 2012 Science Publications

\title{
Functional Recovery of Upper Limb Post-Stroke: Mental Practice with Motor and Non-Motor Imagery
}

\author{
${ }^{1}$ Aline Furtado Bastos, ${ }^{2}$ Beatriz Cantanhede Carrapatoso, \\ ${ }^{3,4}$ Marco Orsini, ${ }^{3}$ Marco Antonio Araujo Leite, \\ ${ }^{4,5}$ Julio Guilherme da Silva and ${ }^{6}$ Gabriela Guerra Leal Souza \\ ${ }^{1}$ Institute of Biophysics Carlos Chagas Filho, \\ Federal University of Rio de Janeiro (UFRJ), Rio de Janeiro, Brazil \\ ${ }^{2}$ Faculty of Physiotherapy, \\ Centro Universitario Serra dos Orgaos (UNIFESO), Rio de Janeiro, Brazil \\ ${ }^{3}$ Departament of Neurology, Movement Disorders Unit, \\ Federal Fluminense University (UFF), Rio de Janeiro, Brazil \\ ${ }^{4}$ Masters Program in Science Rehabilitation, \\ Centro Universitario Augusto Motta (UNISUAM), Rio de Janeiro, Brazil \\ ${ }^{5}$ Department of Medical Clinic, Faculty of Medicine, School of Physiotherapy, \\ Federal University of Rio de Janeiro (UFRJ), Rio de Janeiro, Brazil \\ ${ }^{6}$ Department of Biological Sciences, \\ Federal University of Ouro Preto (UFOP), Minas Gerais, Brazi
}

\begin{abstract}
Problem statement: Mental Practice (MP) with motor imagery consists of a method of training in which motor imagery is used to improve performance. The aim of this study was to investigate the efficacy of MP with motor imagery on motor recovery of the paretic upper limb of poststroke patients in comparison with MP with non-motor imagery. Approach: Four post-stroke patients were divided into two groups: experimental (the physical practice of motor tasks and MP of the same tasks: $n=2$ ) and control (the physical practice of motor tasks and MP of non-motor tasks: $n=2$ ). We evaluated: Heart Rate (HR) and systolic (SBP) and Diastolic Blood Pressure (DBP), immediately before and after execution and imagination of the tasks; time of execution and imagination of each task; and motor function of the paretic upper limb through the questionnaires "Fugl-Meyer for Upper Extremity" and "quality of movement section of arm motor ability test" before and after the 10 weeks of treatment. Results: The qualitative analysis showed that regardless of training, patients spent less time on imagery motor tasks than to execute them; and have increased in HR, SBP and DBP after execution as well as after imagery of motor tasks. The training caused a reduction in the time of execution of motor tasks in both groups and increment on the motor function of the paretic upper limb only in the experimental group. Conclusion: These results suggest that an MP with motor imagery may be an effective method for the functional recovery of the paretic upper limb of post-stroke patients.
\end{abstract}

Key words: Motor imagery, mental practice, rehabilitation, stroke, hemiparesis

\section{INTRODUCTION}

Motor skills require planning and programming before any physical practice. The motor system is responsible not only for production but also by encoding the representational aspects of the movement, for example, the ability to mentally simulate an action. Motor Imagery (MI) originates from an internal model of movement, resulting from mental operations responsible for generating sequential actions or recalling from procedural memory in which the motor program is stored. In this context, MI is usually defined as a dynamic state during which the representation of a given motor act is internally rehearsed in working memory without any overt motor output process (Jeannerod and Decety, 1995; Sirigu and Duhamel, 2001).

There are two different strategies of MI, visual and kinesthetic. Kinesthetic MI is performed in first person,

Corresponding Author: Aline Furtado Bastos, Institute of Biophysics Carlos Chagas Filho, Federal University of Rio de Janeiro (UFRJ), Rio de Janeiro, Brazil 
which performer approximates a real life situation in such a way that the person actually experiences the sensory sensations, providing the feeling of movements as if they were really performed. Indeed, it requires an approximation of the real life phenomenology such that the person actually imagines being inside his/her body and experiencing those sensations that might be expected in the actual situation. On the other hand, visual MI is performed in third person (related to the scenes outside the person) providing the mental visualization of the movement performance as from a distance, actually the subject views himself from the perspective of an external observer (Dickstein and Deutsch, 2007; Stecklow et al., 2007).

Studies have shown that visual and kinesthetic-MI activate different overlapping networks in different situations. Sirigu and Duhamel (2001) showed that healthy subjects were faster to perform kinesthetic MI tasks than visual MI tasks. Similarly patients with inferior temporal lesion were also faster to perform kinesthetic MI task. On the other hand, patients with parietal lesion were faster to perform visual MI task. These results suggest that kinesthetic MI activate primarily motor circuitry and visual MI activate primarily visual circuitry. Other authors reported that kinesthetic-MI activates somatossensory cortex, left inferior parietal lobule, supplementary motor area, precentral gyrus, precuneus, occipitotemporal junction (Ruby and Decety, 2001), frontal cortex, basal ganglia and cerebellum (Guillot et al., 2009). Nevertheless, visual-MI activates occipital and superior parietal regions (Guillot et al., 2009). By the fact that these two modalities generate different patterns of cortical activation, Fery (2003) proposed that using MI to perform a task, the visual strategy is best to tasks based on shape, while the kinesthetic strategy is best to tasks based on time and coordination of the movements.

Mental Practice (MP) consists of a method of training in which $\mathrm{MI}$ is used to improve motor performance (Page et al., 2007). Nevertheless, many questions remain unclear such as the functional recovery of non-trained tasks (Dijkerman et al., 2004; Vries and Mulder, 2006), age, lesion site, cognitive functioning, treatment duration (how many days per week and how many weeks), duration of each session, the modality of MI and the time since onset of motor impairment (acute, subacute or chronic) (Verbunt et al., 2008).

The aim of this study was to investigate the efficacy of mental practice with kinesthetic motor imagery in the motor recovery of upper limb in poststroke patients in comparison with mental practice with non-motor imagery (i.e., training using imagery of static visual scenes that not involve motor simulation nor in first either in third person).

\section{MATERIALS AND METHODS}

Subjects: We analyzed the record of all hemiparetic posts-stroke patients $(\mathrm{n}=75)$, that were in treatment at the Clinic School of Physiotherapy of the Centro Universitário Serra dos Órgãos (UNIFESO) in Rio de Janeiro (Brazil). Inclusion criteria: no more than one stroke; stroke experienced > 5 months before study enrollment; age > 45 and < 80 years old; upper limb strength $\geq$ ' 3 ' on Manual Muscle Strength Scale. Exclusion criteria: excessive spasticity, defined as a score of '3' on the Modified Ashworth Spasticity Scale (Bohannon and Smith, 1987); superior and/or inferior parietal area damage shown on Magnetic Ressonance; orthopaedic, reumathologic or muskuloskeletal damage on paretic upper limb; severe cognitive deficit shown on Mini-mental state examination; motor, sensitive and/or mixed aphasia.

Due to the strict selection criteria adopted in our study, only 4 patients were selected. The experimental protocol was approved by the local Ethical Committee and all volunteers signed an informed consent before starting the treatment.

Outcome measures: Heart Rate (HR) was collected using the Hand-held PalmSat NONIN 2500 oximeter. Systolic (SBP) and Diastolic Blood Pressure (DBP) were collected through auscultatory method using Welch Allyn ADC Durashock Shock Resistant Multicuff aneroid sphygmomanometer with Littmann Master Classic II stethoscope. The time spent on each task was measured using a digital Casio HS-30WN1V chronometer. The functionality of upper limb was register through the questionnaires: "Fugl-Meyer for Upper Extremity" (Maki et al., 2006) and "Quality of movement section of Arm Motor Ability Test" (Morlin et al., 2006).

Procedure: We drew lots and put each patient randomly into one of the two groups: experimental $(\mathrm{n}=$ $2)$ and control $(n=2)$. For both groups the intervention consisted of 1-hour training sessions, once a week for 10 consecutive weeks. All patients were still enrolled in their habitual physical rehabilitation 1 hour once a week, in which not involved any activity to upper limbs. Patients in the experimental group received training sessions that consisted of physical practice of 5 motor tasks plus MP of the same tasks, trough kinesthetic modality (Table 1). Patients in the control group received training sessions that consisted of physical practice of the same 5 motor tasks used in the experimental group plus MP of 5 non-motor tasks, i.e., imagery of static visual scenes (Table 1). 
During the first four weeks, the patients performed five series of physical practice and of MP per session. Each series of physical practice consisted of the execution of each task 10 times and, each series of MP consisted of the imagination of each task 10 times. There was an interval of $5 \mathrm{~min}$ between physical practice and MP. The patient had to indicate the beginning of each task saying "GO" and the end of each task saying "GO".

After four weeks of training, some patients reported pain on the paretic upper limb, therefore we reduced the number of series to 3 times and included series of elongation before and after the protocol. The series of elongation consisted of 3 series of flexibilities exercises for anterior muscles of paretic upper limb that spent $30 \mathrm{sec}$, with $15 \mathrm{sec}$ of interval between each one.

HR, SBP and, DBP were assessed immediately before and after execution and imagination of all tasks during each session. Moreover, the time spent to execute and imagine each task and, the functionality of paretic upper limb were assessed before and after the training program, the latter using "Fugl-Meyer for Upper Extremity" and "Quality of movement section" of "Arm Motor Ability Test".

Instruments: The Fugl-Meyer Scale (Maki et al., 2006) assesses several dimensions of impairment, including range of motion, pain, sensation, upper extremity, lower extremity and balance. The specific items in the upper-extremity subsections were derived from the Brunnström stages of poststroke motor recovery. The data arise from a 3-point ordinal scale (0cannot perform, 1-can perform partially, 2-can perform fully) applied to each item and the items are summed to provide a maximum score of 226 . The upper-extremity motor component, which consists of 66 points, was used in this study.

"Arm Motor Ability Test" (Morlin et al., 2006) is an instrument for assessing qualitative deficits in activities daily of life with upper limb of post-stroke patients. It consists of 13 tasks that reproduce daily activities as measured by a scale ranging from $0-5$ on 'functional hability' and 'quality of movement' ( 0 -do not have, (1) very poor (2) poor (3) average, (4) almost normal and (5) normal). The time spent on each task can range from $60-120 \mathrm{sec}$. Here, we used only the 'Quality of movement' section of this instrument.

Table 1: Motor tasks executed for both groups and imagined for experimental group; non-motor tasks imagined for control group

Motor tasks Non-motor tasks

Pick up A phone on a table in the living room

Reach for and grasping a glass A glass on the sink in the kitchen

and take it to the mouth

Turn a page of a book

A toothbrush in the cabinet in the bathroom

Reach the top of the head

A television on the bookcase in the living room

Open and close a door

\section{RESULTS}

Applying the aforementioned study criteria, four subjects were included in this study. See Table 2 to group characteristics.

The time spent to execute motor tasks reduced $14.81 \%$ and $21.39 \%$ after 10 weeks of treatment, for experimental and control groups respectively. For the simulation of motor tasks, the experimental group showed an increase of $3.72 \%$ after training. The comparison between time spent to execution and simulation of motor tasks showed that, regardless on training, the experimental group spent less time to mentally simulate than to perform motor tasks (mean difference of $33.64 \%$ at baseline and $19.20 \%$ after training). For the mental simulation of non-motor tasks, control group diminished the time spent in $7.75 \%$ (Table 3 ).

The assessment of HR in the experimental group showed an increase of $1.57 \%$ after the execution of motor tasks and an increase of $1.30 \%$ after the mental simulation of the same tasks. In the control group, the execution of motor tasks caused an increase of $10.72 \%$ while the mental simulation of non-motor tasks caused a reduction of $2.04 \%$ (Table 4).

Concerning the SBP, the execution of motor tasks in the experimental group caused an increase of $4.13 \%$ and mental simulation of the same tasks caused an increase of $1.17 \%$. In the control group, there was an increase of $4.97 \%$ after the execution of motor tasks and a decrease of $1.45 \%$ after the simulation of nonmotor tasks (Table 4).

Table 2: Group characteristics

\begin{tabular}{lll}
\hline & Experimental group & Control group \\
\hline Men / Women & Feb-00 & Feb-00 \\
Age (years) & $60 \pm 15$ & $62 \pm 24$ \\
Time Post-stroke (months) & $55.5 \pm 48.8$ & $11 \pm 8.5$ \\
Arm Affected (R/L) & Feb-00 & Feb-00 \\
\hline
\end{tabular}

$\mathrm{R}=$ Right; $\mathrm{L}=$ Left

Table 3: Variation of the time spent and the function of the paretic upper limb, before and after treatment, for the execution and simulation of the tasks, in experimental and control groups

\begin{tabular}{lcr} 
& Baseline & Post intervention \\
\hline Cronometry of physical practice (s) & & \\
Experimental & 6.55 & 5.58 \\
Control & 10.51 & 8.26 \\
Cronometry of mental practice (s) & & \\
Experimental & 4.35 & 4.51 \\
Control & 2.58 & 2.39 \\
FM & & \\
Experimental & 46.00 & 47.00 \\
Control & 42.00 & 37.00 \\
AMAT & & \\
Experimental & 110.00 & 117.00 \\
Control & 104.00 & 100.00 \\
\hline S = Seconds; FM=Fugl-Meyer for Upper Extremity; ARAT=Arm \\
Motor Ability Test (Quality of Movement Section) \\
$\quad$
\end{tabular}


Table 4: Mean Heart Rate and Systolic and Diastolic Blood Pressure immediately before and after the execution and mental simulation of the five tasks during the 10 sessions of training in experimental and control groups

\begin{tabular}{lllll}
\hline & Execution & & Simulation & \\
& Pre & Post & Pre & Post \\
\hline HR (bpm) & & & & \\
Experimental & 89 & 91 & 89 & 90 \\
Control & 78 & 87 & 76 & 74 \\
SBP (mmHg) & & & & 121 \\
Experimental & 119 & 124 & 119 & 115 \\
Control & 117 & 122 & 117 & 87 \\
DBP (mmHg) & & & & 77 \\
$\quad$ Experimental & 84 & 87 & 86 & 78 \\
Control & 77 & 80 & 78 & \\
\hline
\end{tabular}

HR = Heart Rate; bpm = beats per minute; SBP = Systolic Blood Pressure; $\mathrm{mmHg}=$ millimeters of mercury; $\mathrm{DBP}=$ Diastolic Blood Pressure

In relation to DBP, in the experimental group, the execution of motor tasks caused an increase of $3.20 \%$ and mental simulation of the same tasks caused an increase of $1.28 \%$. In the control group, there was an increase of $3.23 \%$ in DBP after the execution of motor tasks and a decrease of $1.93 \%$ after the simulation of non-motor tasks (Table 4).

Take into consideration the "Fugl-Meyer for Upper Extremity", the training program caused an increase of $2.17 \%$ in the experimental group and a decrease of $10.84 \%$. Regarding the "quality of movement section" of "Arm Motor Ability Test", the experimental group increased the score in $6.36 \%$ and the control group decreased in $3.85 \%$ (Table 3 ).

\section{DISCUSSION}

This study used a protocol of kinesthetic motor imagery associated with physical practice of activities of daily living to treat motor deficits of upper limb in post-stroke patients. Our results showed a reduction in the time spent for execution of motor tasks. In the same vein, as shown by Gentili et al. (2006), in a study with healthy subjects, after both physical practice and MP of an upper limb task, hand movement duration decreased and peak acceleration increased. Other studies evaluated patients with hemiparesis during MP of lower limb tasks and showed significant increases in gait speed, stride length, cadence and single-support time of the affected lower limb (Dunsky et al., 2008; Hwang et al., 2010).

It was observed a difference between the times of execution and simulation of motor tasks in the experimental group. The patients spent more time to execute than to simulate a motor task and, this difference occurred also before as to after the training program. Corroborating these findings, Mulder et al. (2007) showed that elderly individuals were slightly worse in MI capacity than younger participants, particularly in relation to kinesthetic modality, which was used here. Furthermore, Sharma et al. (2006) reported that midbrain, cerebellum and/or primary motor cortex damages could slow the execution of movements compared to mental simulation of the same. This may indicate that the age and the brain injury of the patients who participated in this study may have contributed to a slower execution of motor tasks without compromise, however, the imagination of them. These findings are consistent with a recent study that evaluated post-stroke patients and found that they spent more time to execute tasks than to imagine them (Wu et al., 2010).

In fact, to the best of our knowledge, this study is the first to demonstrate the autonomic activation on post-stroke patients through MP with motor imagery. In the current study, we showed that the experimental group increased HR, SBP and DBP after execution as well as after simulation of motor tasks compared with baseline. The control group showed an increase in these variables after the execution of motor tasks, however there was a decrease after the simulation of non-motor tasks. Decety et al. (1993) revealed an increase of HR after MI, in healthy subjects. Oishi et al. (2000), which assess eight skaters, have also observed an increase of HR after MI. This supports the idea that impaired patients, as well as healthy individuals, could activate the Sympathetic and/or deactivate the Parasympathetic Autonomic Nervous System during imagery of motor tasks, thereby resulting in increasing HR, SBP and DBP. Moreover, non-motor tasks appear to have caused a relaxation of the individuals, since there was a reduction of autonomic variables.

Interestingly, we found an improvement in quality of movement and sensory-motor function in the affected upper limb only in the experimental group. This result is consistent with the results found by different authors through various types of experimental protocols. Some studies used only MP, with no control group to show improvement in the paretic limb function. Dijkerman et al. (2004) and Stevens and Stoykov (2003) showed that patients trained with MP improve the performance of trained motor tasks. Johnson-Frey (2004), using the technique of functional Magnetic Resonance Imaging (fMRI), showed that even with any overt output, mentally simulate motor tasks can facilitate the reorganization of sensorimotor areas of the injured hemisphere.

Other studies used MP and physical practice and compared some measures before and after the training program. They showed that a rehabilitation program for the affected upper extremity incorporating MP appears 
to improve movement via significant cortical reorganization observed by fMRI (Page et al., 2009) or to improve function of affected limb observed by questionnaires (Page et al., 2001; Hewett et al., 2007). There are few studies that used control groups to show the effectiveness of MP on the gain of function. Muller et al. (2007) divided the patients into three treatment groups ((i) MP of a non-sequential finger movement, (ii) physical practice of the same non-sequential task and (iii) conventional physical therapy, that included gross hand movements, like holding a cup) and showed that the MP and the physical practice groups rescued the ability to perform finger movements with a consequent improvement on manual function. Other studies showed that MP associated with physical practice is more effective for improving the function of the affected limb than just the use of relaxation techniques (Page et al., 2005) or than combination of relaxation techniques with physical practice (Page et al., 2007; 2011). Thus, the present study, despite the small sample, has been contributing with the few existing controlled studies. Even with little time of training, seems to have difference between the groups, showing that the physical practice associated with MP of motor tasks through kinesthetic modality may be more effective than physical practice of motor tasks per se. Besides, this study used chronometric, autonomic and functional scales measures in an experimental and control group to demonstrate the effectiveness of the MP with motor imagery associated with physical practice in post-stroke patients.

\section{CONCLUSION}

The present results suggest that training using physical practice plus MP seems to be an effective method for the functional recovery of upper limb of poststroke patients. Thus, it seems interesting to combine the use of MP to treatment with conventional physiotherapy. Future investigations should be conducted to determine the guidelines for the practice of the technique, the hemodynamic and metabolic effects and its application on patients with other motor diseases.

\section{REFERENCES}

Bohannon, R.W. and M.B. Smith, 1987. Interrater reliability of a modified Ashworth scale of muscle spasticity. Phys. Ther., 67: 206-207. PMID: 3809245

Decety, J., M. Jeannerod, D. Durozard and G. Baverel, 1993. Central activation of autonomic effectors during mental simulation of motor actions in man. J. Physiol., 461: 549-563. PMID: 8102402
Dickstein, R. and J.E. Deutsch, 2007. Motor imagery in physical therapist practice. Phys. Ther., 87: 942953. DOI: $10.2522 / p t j .20060331$

Dijkerman, H.C., M. Ietswaart, M. Johnston and R.S. MacWalter, 2004. Does motor imagery training improve hand function in chronic stroke patients? A pilot study. Clin. Rehabil., 18: 538-549. DOI: 10.1191/0269215504cr769oa

Dunsky, A., R. Dickstein, E. Marcovitz, S. Levy and J. Deutsch, 2008. Home-based motor imagery training for gait rehabilitation of people with chronic poststroke hemiparesis. Arch. Phys. Med. Rehabil., 89: 1580-1588. DOI: 10.1016/j.apmr.2007.12.039

Fery, Y.A., 2003. Differentiating visual and kinesthetic imagery in mental practice. Can. J. Exp. Psyc., 57: 1-10. DOI: $10.1037 / \mathrm{h} 0087408$

Gentili, R., C. Papaxanthis and T. Pozzo, 2006. Improvement and generalization of arm motor performance through motor imagery practice. Neuroscience, 137: 761-772. DOI: 10.1016/j.neuroscience.2005.10.013

Guillot, A., C. Collet, V.A. Nguyen, F. Malouin and C. Richards et al., 2009. Brain activity during visual versus kinesthetic imagery: An fMRI study. Hum. Brain Mapp., 30: 2157-2172. DOI: 10.1002/hbm. 20658

Hewett, T.E., K.R. Ford, P. Levine and S.J. Page, 2007. Reaching kinematics to measure motor changes after mental practice in stroke. Top Stroke Rehabil., 14: 23-29. DOI: 10.1310/tsr1404-23

Hwang, S., H.S. Jeon, C.H. Yi, O.Y. Kwon and S.H. Cho et al., 2010. Locomotor imagery training improves gait performance in people with chronic hemiparetic stroke: A controlled clinical trial. Clin. Rehabil., 24: 514-522. DOI: 10.1177/0269215509360640

Jeannerod, M. and J. Decety, 1995. Mental motor imagery: A window into the representational stages of action. Curr. Opin. Neurobiol., 5: 727-732. DOI: 10.1016/0959-4388(95)80099-9

Johnson-Frey, S.H., 2004. Stimulation through simulation? Motor imagery and functional reorganization in hemiplegic stroke patients. Brain Cognit., 55: 328-331. DOI: 10.1016/j.bandc.2004.02.032

Maki, T., E.M.A.B. Quagliato, E.W.A. Cacho, L.P.S. Paz and N.H. Nascimento et al., 2006. Estudo de confiabilidade da aplicação da escala de fuglmeyer no brasil. Rev. Bras. Fisioter., 10: 177-183. DOI: $10.1590 / \mathrm{S} 1413-35552006000200007$ 
Morlin, A.C.G., A.M. Delattre, E.W.A. Cacho, T.D. Oberg and R.D. Oliveira, 2006. Concordância e tradução para o português do Teste de Habilidade Motora do Membro Superior-THMMS. Rev. Neurocienc., 14: 6-9.

Mulder, T., J.B.H. Hochstenbach, M.J.G.V. Heuvelen and A.R.D. Otter et al., 2007. Motor imagery: The relation between age and imagery capacity. Hum. Movement Sci., 26: 203-211. DOI: 10.1016/j.humov.2007.01.001

Muller, K., C.M. Butefischa, R.J. Seitz and V. Homberg, 2007. Mental practice improves hand function after hemiparetic stroke. Restor. Neurol. Neurosci., 25: 501-511. PMID: 18334768

Oishi, K., T. Kasai and T. Maeshima, 2000. Autonomic response specificity during motor imagery. J. Physiol. Anthropol., 19: 255-261. DOI: 10.2114/jpa.19.255

Page, S.J., J.P. Szaflarski, J.C. Eliassen, H. Pan and S.C. Cramer, 2009. Cortical plasticity following motor skill learning during mental practice in stroke. Neurorehabil Neural Repair, 32: 382-388. DOI: $10.1177 / 1545968308326427$

Page, S.J., K. Dunning, V. Hermann, A. Leonard and P. Levine, 2011. Longer versus shorter mental practice sessions for affected upper extremity movement after stroke: A randomized controlled trial. Clin. Rehab., 25: 627-637. DOI: $10.1177 / 0269215510395793$

Page, S.J., P. Levine and A.C. Leonard, 2005. Effects of mental practice on affected limb use and function in chronic stroke. Arch. Phys. Med. Rehabil., 86: 399-402. DOI: 10.1016/j.apmr.2004.10.002

Page, S.J., P. Levine and A.C. Leonard, 2007. Mental practice in chronic stroke. Results of a randomized. Placebo-Controlled Trial. Stroke, 38: 1293-1297. DOI: 10.1161/01.STR.0000260205.67348.2b

Page, S.J., P. Levine, S.A. Sisto and M.V. Johnston, 2001. Mental practice combined with physical practice for upper-limb motor deficit in subacute stroke. Phys. Ther., 81: 1455-1462. PMID: 11509075
Ruby, P. and J. Decety, 2001. Effect of subjective perspective taking during simulation of action: A PET investigation of agency. Nature Neurosci., 4: 546-550. PMID: 11319565

Sharma, N., V.M. Pomeroy and J. Baron, 2006. Motor Imagery: A backdoor to the motor system after stroke? Stroke, 37: 1941-1953. DOI: 10.1161/01.STR.0000226902.43357.fc

Sirigu, A. and J.R. Duhamel, 2001. Motor and visual imagery as two complementary but neurally dissociable mental processes. J. Cog. Neurosci., 13: 910-919. DOI: 10.1162/089892901753165827

Stecklow, M.V., A.F. Catelli and M. Cagy, 2007. Alterações na banda alfa do eletrencefalograma durante imagetica motora visual e cinestesica. Arq Neuropsiquiatr, 65: 1084-1088. DOI: 10.1590/S0004-282X2007000600034

Stevens, J.A. and M.E.P. Stoykov, 2003. Using motor imagery in the rehabilitation of hemiparesis. Arch. Phys. Med. Rehabil., 84: 1090-1092. DOI: 10.1016/S0003-9993(03)00042-X

Verbunt, J.A., H.A.M. Seelen, F.P. Ramos, B.H.M. Michielsen and W.L. Wetzelaer et al., 2008. Mental practice-based rehabilitation training to improve arm function and daily activity performance in stroke patients: A randomized clinical trial. BMC Neurology, 8: 1-10. DOI: 10.1186/1471-2377-8-7

Vries, S. and T. Mulder, 2006. Motor imagery and stroke rehabilitation: A critical discussion. J. Rehabil. Med., 39: 1-9. PMID: 17225031

Wu, A.J., V. Hermann, J. Ying and S.J. Page, 2010. Chronometry of mentally versus physically practiced tasks in people with stroke. Am. J. Occup. Ther., 64: 929-34. DOI: 10.5014/ajot.2010.09005 